\title{
Effectiveness of Dioscorea hispida Dennst as antibacterial and antibiofilm agent
}

\author{
Nur Hazlin Hazrin-Chong ${ }^{{ }^{*}}$, Ahmad Muzamil Azeem¹, Nik Nor Imam Nik Mat Zin¹, Mohamad Azwani Shah Mat \\ Lazim $^{2}$ \\ ${ }^{1}$ School of Biosciences and Biotechnology, Faculty of Science and Technology, Universiti Kebangsaan Malaysia, 43600 \\ Bangi, Selangor, Malaysia. \\ ${ }^{2}$ School of Chemical Sciences and Technology, Faculty of Science and Technology, Universiti Kebangsaan Malaysia, \\ 43600 Bangi, Selangor, Malaysia. \\ Email: hazlin@ukm.edu.my
}

\begin{abstract}
Aims: Antimicrobial resistance (AMR) is a growing threat to public health, where treatments using conventional drugs are becoming ineffective. One viable but underexplored alternative is through the use of Dioscorea hispida, a wild plant that exhibits antimicrobial properties. This study aims to explore $D$. hispida effectiveness as an antibacterial and antibiofilm agent against selected pathogenic and non-pathogenic bacteria.

Methodology and results: Different concentrations of $D$. hispida crude extracts $(0-2.5 \mathrm{mg} / \mathrm{mL})$ were tested against the growth of planktonic bacterial cells over $24 \mathrm{~h}$ incubation, and the half maximal effective concentration $\left(E_{50}\right)$ obtained was used in the antibiofilm test over 24 and $48 \mathrm{~h}$. All bacteria treated with $D$. hispida showed significant $(\mathrm{P}<0.05)$ reduction in planktonic cell and biofilm densities against the negative control starting at $0.3 \mathrm{mg} / \mathrm{mL}$. However, in comparison to the antibiotic, only certain bacteria were significantly affected by $D$. hispida, implying the plant has a 'moderate' biocidal activity in general. Furthermore, Atomic Force Microscopy imaging of $S$. aureus biofilm with $D$. hispida revealed increased height and width of cell clusters despite reduction in volume compared to the negative control, suggesting unique biofilm resistance behaviour against the plant.

Conclusion, significance and impact of study: This study demonstrated $D$. hispida capability as a natural antimicrobial and antibiofilm agent. The plant could complement current antimicrobials to maximise killing efficiency and minimise occurrences of resistance. Unique biofilm behaviour against $D$. hispida also warrants further investigation on the effect of biocides towards biofilm structure. Overall, this research provides new insights into a traditional plant-based antimicrobial activity in combating infectious diseases and AMR.
\end{abstract}

Keywords: Dioscorea hispida, antimicrobial, biofilm, traditional medicine, Atomic Force Microscopy

\section{INTRODUCTION}

Infections and diseases caused by microorganisms are a constant threat to the society, requiring medical intervention including the application of antimicrobial drugs (e.g. antibiotics, antifungals and antivirals). However, the misuse and overuse of these antimicrobials have given rise to a number of antimicrobial resistant (AMR) microorganisms, leading to the evolution of "superbugs". These superbugs, such as those coming from a group coined "ESKAPE" (Enterococcus faecium, Staphylococcus aureus, Klebsiella pneumoniae, Acinetobacter baumanii, Pseudomonas aeruginosa and Enterobacter species) are multidrug resistant, causing serious consequences to public health particularly to the more vulnerable groups within society (e.g. small children, elderly and immune-compromised patients). This phenomenon is predicted to worsen due to the uncontrolled use of antimicrobials and the rapid capability of these bacteria to develop resistance (Unemo and
Jensen, 2017). To combat this problem, developing viable alternatives to the commonly used antimicrobials is necessary apart from efforts to control antimicrobial over prescription and consumption.

Dioscorea hispida Dennst, or locally known as Ubi Gadong, is a promising natural alternative to the commonly used antimicrobials. $D$. hispida are edible wild tubers that are currently under-exploited but potentially effective as an antimicrobial due to their high content of bioactive alkaloids (Hron et al., 1997). These alkaloids (e.g. dioscorine, found in the rhizome) are poisonous and therefore, they are removed from the plant prior to consumption. Despite the toxicity, $D$. hispida is primarily consumed in some tropical regions and used as a therapeutic drug to alleviate inflammation and combat indigestion (Mat Lazim et al., 2016). Miah et al. (2018) has recently shown that certain fractions of phenolic compounds in $D$. hispida possess high antioxidant and 
Malays. J. Microbiol. Vol 14(6) Special Issue 2018, pp. 462-467 DOI: http://dx.doi.org/10.21161/mjm.1461803

thrombolytic activities, making the plant a potent traditional therapeutic. The free radical scavenging property in $D$. hispida was also observed in other Dioscorea species, e.g. D. batatas, that was linked to its dioscorine content (Hou et al., 2001; Ohizumi et al., 2009).

Previous studies have demonstrated $D$. hispida as a potential antimicrobial agent and insecticide whilst showing acceptable tolerance in vertebrates (Bhamarapravati et al., 2003; Otake et al., 1995). The starch was tested as one of the disinfectants against Helicobacter pylori, a Gram-negative bacterium that causes several gut diseases including stomach ulcer (Bhamarapravati et al., 2003). Furthermore, through disk diffusion tests, $D$. hispida showed the ability to inhibit the growth of certain groups of bacteria and fungi (Azman et al., 2015; Miah et al., 2018). Dioscorea hispida has also been used as a coating material on rubber wood and demonstrated repelling activity against white-rot fungi and termites (Mat Lazim et al., 2016). Interestingly, toxicity tests against zebrafish as a vertebrate model showed the organism's tolerance towards the starch at an acceptable concentration, implying the suitability of $D$. hispida as an antimicrobial agent with little negative impact to vertebrates (Azman et al., 2015).

Whilst the above studies on D. hispida are useful, there is still a large gap in knowledge and understanding to establish $D$. hispida as an effective antimicrobial agent. For example, the maximal effective concentration (EC) of $D$. hispida against bacteria was not determined from the limited range of concentrations tested in previous studies. Additionally, it has not been established whether $D$. hispida effectively targets a broad range of bacteria based on the limited number of species used. Furthermore, the potential use of $D$. hispida as an antibiofilm agent has never been explored. Biofilms are known to be more resistant towards environmental stress (e.g. starvation, toxicity, predation) than its free-living, planktonic form and have been the root cause of many infectious diseases (Donlan and Costerton, 2002). Therefore, including biofilms as part of antimicrobial research is imperative. This study explored the effectiveness of $D$. hispida as an antibacterial and antibiofilm agent by testing the plant starch against selected microorganisms in their planktonic and biofilm forms. Further, through Atomic Force Microscopy, the biofilm morphology of a bacterium in the presence of $D$. hispida was compared against that of control biofilms without $D$. hispida or with an antibiotic.

\section{MATERIALS AND METHODS}

\section{Discorea hispida starch and liquid stock preparation}

Starch extraction was performed according to method described by Mat Lazim et al. (2016), in which the wild tuber was washed, pulverised, separated and dried. To obtain liquid stock of $D$. hispida $(50 \mathrm{mg} / \mathrm{mL})$, the prepared starch $(50 \mathrm{~g})$ was mixed with $100 \mathrm{~mL} 5 \%(\mathrm{w} / \mathrm{v}) \mathrm{NaOH}$, with agitation at $200 \mathrm{rpm}$ for $2 \mathrm{~h}$.

\section{D. hispida antibacterial test against planktonic bacterial cultures}

Escherichia coli UKMCC1006, Staphylococcus aureus UKMCC1016, Pseudomonas aeruginosa UKMCC1011, Klebsiella pneumoniae UKMCC1007 and Bacillus subtilis UKMCC1002 (obtained from the Universiti Kebangsaan Malaysia Culture Collection, Bangi, Malaysia), both in free-living (planktonic) and biofilm forms were used to test the effectiveness of $D$. hispida starch as an antibacterial agent.

Overnight bacterial cultures were prepared by incubation in Nutrient Broth (NB) (per $\mathrm{L}$ of $\mathrm{dH}_{2} \mathrm{O}: 5 \mathrm{~g}$ peptone and $3 \mathrm{~g}$ meat extract, $\mathrm{pH} 7$ ) at $37^{\circ} \mathrm{C}$. Bacterial cells were diluted to approximately $10^{7}$ cells $/ \mathrm{mL}$ before being tested against $D$. hispida at the following concentrations $(\mathrm{mg} / \mathrm{mL}): 0.16,0.31,0.63,1.25$ and 2.5 for $24 \mathrm{~h}$ at $37^{\circ} \mathrm{C}$. Cell growth inhibition was measured by adding 3-(4,5-dimethylthiazol-2-yl)-2,5-diphenyltetrazoliu$\mathrm{m}$ bromide (MTT) at a ratio of 2:15 (MTT: cell culture) before incubating at $37^{\circ} \mathrm{C}$ for $1 \mathrm{~h}$ and Optical Density (OD) was measured at $595 \mathrm{~nm}$. Gentamicin $(150 \mu \mathrm{g} / \mathrm{mL})$ served as the positive control for inhibition, whilst bacterial cultures only (i.e. without $D$. hispida) were used as a negative control. All tests were conducted in at least three independent replicates and three technical replicates for each bacterium.

The half maximal effective concentration $\left(E_{50}\right.$, i.e. that of which inhibits $50 \%$ of the bacterial population) of $D$. hispida was determined through plotting the percentage of bacterial inhibition against $D$. hispida concentration using the GraphPad Prism 7 software. The $E_{50}$ value obtained for each bacterium was then used in the antibiofilm experiment. The percentage of bacterial inhibition was calculated using the following formula:

$$
O D_{\text {sample }}-O D_{\text {extract }} / O D_{\text {control }} \times 100
$$

where $\mathrm{OD}_{\text {sample }}=\mathrm{OD}$ of the tested bacterial culture with $D$. hispida at a specific concentration, $\mathrm{OD}_{\text {extract }}=\mathrm{OD}$ of $D$. hispida at concentration used in ODsample without bacteria and $\mathrm{OD}_{\text {control }}=\mathrm{OD}$ of bacteria without $D$. hispida but with $\mathrm{NaOH}$ (used as solvent for $D$. hispida) at concentration used in $\mathrm{OD}_{\text {sample. }}$

\section{D. hispida treatment against bacterial biofilms}

Biofilm for each bacterium was prepared and its biomass quantified according to the previously described method by O'Toole (2011), with or without the addition of $D$. hispida. An overnight culture of each bacterium was prepared in NB at $37{ }^{\circ} \mathrm{C}$ and inoculated (1:100 dilution) into fresh $\mathrm{M} 9$ medium (per $\mathrm{L}$ of $\mathrm{dH}_{2} \mathrm{O}: 64 \mathrm{~g} \mathrm{Na}_{2} \mathrm{HPO}_{4}$ $7 \mathrm{H}_{2} \mathrm{O}, 15 \mathrm{~g} \mathrm{KH}_{2} \mathrm{PO}_{4}, 2.5 \mathrm{~g} \mathrm{NaCl}, 5.0 \mathrm{~g} \mathrm{NH}_{4} \mathrm{Cl}$, $2 \mathrm{~mL}$ of $1 \mathrm{M}$ $\mathrm{MgSO}_{4}, 100 \mu \mathrm{L}$ of $1 \mathrm{M} \mathrm{CaCl}_{2}$ and $20 \mathrm{~mL}$ of $20 \%(\mathrm{w} / \mathrm{v}) \mathrm{D}$ glucose solution) with $0.25 \%(\mathrm{w} / \mathrm{v})$ of casamino acids (CAA). D. hispida was added into each bacterial culture at $E_{50}$ previously obtained from the antibacterial test and the culture was incubated at $37{ }^{\circ} \mathrm{C}$ for 24 and $48 \mathrm{~h}$. Biofilm density for each bacterium was measured at 550 
Malays. J. Microbiol. Vol 14(6) Special Issue 2018, pp. 462-467 DOI: http://dx.doi.org/10.21161/mjm.1461803

$\mathrm{nm}$ using Crystal Violet staining. Gentamicin $(150 \mu \mathrm{g} / \mathrm{mL})$ was used as positive control, whilst negative control contained zero amount of $D$. hispida starch. All tests were conducted in at least three independent replicates and three technical replicates for each bacterium.

\section{Atomic Force Microscopy (AFM) analysis of S. aureus} biofilm in the presence of $D$. hispida

The effect of $D$. hispida against a bacterial biofilm was further observed using AFM. S. aureus was chosen due to its pathogenicity and relatively higher susceptibility to $D$. hispida than other tested bacteria based on the biofilm assay. Mica sheets $(1 \mathrm{~cm} \times 1 \mathrm{~cm}$ each, used as base for biofilm), were sterilised in $0.5 \% \mathrm{NaOCl}_{3}$ for $2 \mathrm{~h}$ and rinsed 3 times with sterile distilled water prior to putting each into a well of a 12-well plate that contained M9 media with glucose and CAA. An overnight culture of $S$. aureus was inoculated $(1: 100)$ into a well containing the M9 medium, mica sheet and $D$. hispida at 0 or $2.5 \mathrm{mg} / \mathrm{mL}$ (the highest concentration used in this study). Streptomycin (150 $\mu \mathrm{g} / \mathrm{mL}$ ) was used as positive control in replacement of $D$. hispida. The cultures were incubated at $37^{\circ} \mathrm{C}$ for $24 \mathrm{~h}$ in stagnation with at least three independent replicates for each test.

After incubation, the mica sheets containing the biofilms were gently rinsed in sterile M9 media three times before air-dried in a laminar flow cabinet for $4 \mathrm{~h}$ at room temperature. Each biofilm was visualised using an atomic force microscope (Dimension V, Veeco Instruments Inc., New York) operated by the Research NanoScope 7.20 software (Veeco Instruments Inc., New York). The cantilever used had nominal spring constants between 20-80 $\mathrm{N} \mathrm{m}^{-1}$ and frequency of $335 \mathrm{kHz}$. Scans were conducted in tapping mode at $20 \times 20 \mu \mathrm{m}$. At least three scans per independent replicate was performed for each sample. Further data analysis was conducted using Gwyddion version 2.5 software (Nečas and Klapetek, 2012).

\section{RESULTS AND DISCUSSION}

In this study, we explored the effectiveness of $D$. hispida as an antibacterial and antibiofilm agent, serving as an alternative to the over prescribed and over consumed antimicrobials such as antibiotics. The quest for an effective substitute is timely due to the emergence of multidrug resistant microorganisms such as the 'ESKAPE' pathogens, partly caused by an increasing dependency on the same class of drugs to treat infections. Dioscorea hispida Dennst is a wild, tuberous plant available in Malaysia and Southeast Asia and its known toxicity makes it of high potential as a locally sourced biocide. As shown in this study and past research, the tuber was able to target a considerable range of microorganisms and invertebrates, whilst showing an acceptable tolerance level in vertebrates. Here, we elaborated further the effect of $D$. hispida against a selected group of bacteria that are Gram negative (E. coli, $P$. aeruginosa and $K$. pneumoniae) and Gram positive ( $S$. aureus and $B$. subtilis) in both planktonic and biofilm forms.

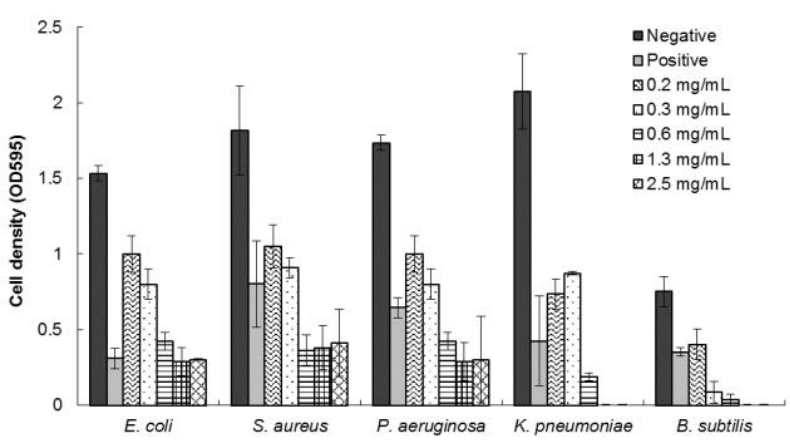

Figure 1: Effect of different $D$. hispida concentrations $(0.2,0.3,0.6,1.3$ and $2.5 \mathrm{mg} / \mathrm{mL})$ on planktonic cell density (OD595) of E. coli, S. aureus, $P$. aeruginosa, $K$. pneumoniae and $B$. subtilis at $24 \mathrm{~h}$ of incubation. Gentamicin $(150 \mu \mathrm{g} / \mathrm{mL})$ was used as positive control. All tests were conducted in three independent replicates.

Table 1: Half maximal effective concentration $\left(E_{50}\right)$ of $D$. hispida against $E$. coli, $S$. aureus, $P$. aeruginosa, $K$. pneumoniae and $B$. subtilis.

\begin{tabular}{ll}
\hline Bacteria & $\mathrm{EC}_{50}(\mathrm{mg} / \mathrm{mL})$ \\
\hline E. coli & $0.4226 \pm 0.08$ \\
P. aeruginosa & $0.4088 \pm 0.09$ \\
K. pneumoniae & $0.4223 \pm 0.04$ \\
S. aureus & $0.3101 \pm 0.02$ \\
B. subtilis & $0.3041 \pm 0.04$ \\
\hline
\end{tabular}

Overall, the presence of $D$. hispida significantly $(\mathrm{P}<0.05)$ reduced bacterial growth compared to those without any treatment. This was clearly shown by the decrease in bacterial cell density (OD595) as $D$. hispida concentration increased after $24 \mathrm{~h}$ of incubation (Figure 1). At the lowest $D$. hispida concentration $(0.2 \mathrm{mg} / \mathrm{mL})$, all five microorganisms showed approximately two-third to half the cell density of negative control (i.e. without $D$. hispida). Cell density was further reduced to more than half at $0.6 \mathrm{mg} / \mathrm{mL} D$. hispida for all bacteria. However, as $D$. hispida concentration increased further $(>0.6 \mathrm{mg} / \mathrm{mL})$, the cell density remained relatively constant for $E$. coli, $S$. aureus and $P$. aeruginosa but declined to almost zero for $K$. pneumoniae and $B$. subtilis, implying higher resistance in the former bacterial group than the latter. The varied susceptibility amongst bacteria towards $D$. hispida is expected and in line with previous findings, where the starch worked more effectively against certain bacteria over others (Azman et al., 2015; Miah et al., 2018). The mechanism of action by $D$. hispida against microorganisms is still largely unknown; however, the tuber contains a high amount of phenolic compounds that have been previously demonstrated to be antibacterial based on a number of mechanisms, including membrane permeabilisation and disruption, redox imbalance 
induction and catalase inhibition (Taylor et al., 2004; Greenberg et al., 2008; Araya-Cloutier et al., 2018; Ajiboye et al., 2018; Carvalho et al., 2018). Thus, phenolics such as the alkaloid dioscorine found in $D$. hispida may work in a similar manner to that of other phenolic compounds, but further research into $D$. hispida mechanism of action is necessary to ascertain this. Interestingly, the two bacteria (K. pneumoniae and $B$. subtilis) in which $D$. hispida was most effective against were Gram negative and positive, respectively, therefore implying that cell wall variation holds little importance to the starch mode of action.

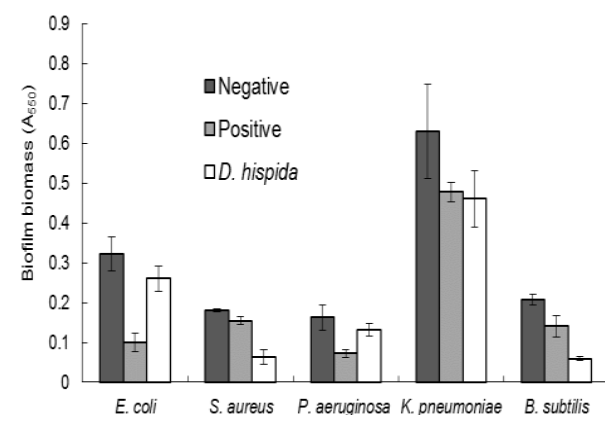

$24 \mathrm{~h}$

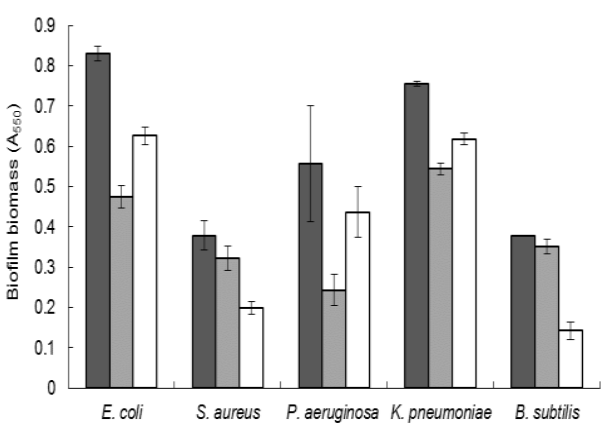

$48 \mathrm{~h}$

Figure 2: Effect of $D$. hispida (at $\mathrm{EC}_{50}$ ) on biofilm biomass $\left(\mathrm{OD}_{550}\right)$ of $E$. coli, $S$. aureus, $P$. aeruginosa, $K$. pneumoniae and $B$. subtilis at 24 and $48 \mathrm{~h}$ of incubation. All microorganisms showed significant $(P<0.05)$ reduction in biofilm density against the negative control (without $D$. hispida), however, performance against the positive control (150 $\mu \mathrm{g} / \mathrm{mL}$ gentamicin) varied. All tests were conducted in three independent replicates.

Despite the significant reduction in bacterial cell density with $D$. hispida, a higher dosage of the starch (i.e. $>0.6 \mathrm{mg} / \mathrm{mL}$ ) was still necessary to render it as or more effective than the antibiotic control. From the results shown (Table 1), D. hispida needed to be at least 0.3 $\mathrm{mg} / \mathrm{mL}$ for $B$. subtilis and $0.6 \mathrm{mg} / \mathrm{mL}$ for other bacteria to be significantly more effective than gentamicin at 0.15 $\mathrm{mg} / \mathrm{mL}(\mathrm{P}<0.05)$. This shows $D$. hispida has a 'moderate' antibacterial activity compared to antibiotics, which are still more potent than the starch. Miah et al. (2018) showed similar trend (where antibiotics outperform $D$. hispida) through disk diffusion tests of $D$. hispida phenolic extracts $(400 \mu \mathrm{g} / \mathrm{disc})$ and ciprofloxacin (30 $\mathrm{\mu g} / \mathrm{disc})$ against a number of pathogenic bacteria. Although $D$. hispida is effective as an antibacterial agent (despite its weaker performance than antibiotics), the plant could also act as a complement to antimicrobials to achieve maximum killing of pathogens and minimise the emergence of resistant microorganisms. However, the question of whether or not the performance of the antibiotic with $D$. hispida is more effective than that of the antibiotic alone still needs to be addressed with further research.

The effectiveness of $D$. hispida was explored further by observing its impact on biofilms. In general, microorganisms are able to grow as two different phenotypes, i.e. 1) planktonic, free-living cells; or 2) biofilms, where cells congregate and are attached to a surface or each other, encased by a layer of extracellular polymer matrix. Biofilms have been shown to be the more predominant form than planktonic cells in causing infections due to their higher resistance to antimicrobials and other environmental stresses. The sessile lifestyle also increases the microorganism's capability to invade host cells, which also, together with host extracellular matrix proteins, act as substrata for microbial cell attachment (Bjarnsholt, 2013; Høiby et al., 2010). For examples, $P$. aeruginosa biofilm has been known to be the causative agent for lung infection (pneumonia) in cystic fibrosis patients, whereas $S$. aureus in its sessile form causes nosocomial infection, particularly from the contamination of medical devices (e.g. catheters, tubes) (Gellatly and Hancock, 2013; Lister and Horswill, 2014). Thus, it is of paramount importance that the biofilm form, apart from the cell free-living lifestyle, is addressed in this study. Biofilm biomass was quantified after 24 and $48 \mathrm{~h}$ of incubation with half maximal concentration of $D$. hispida obtained from the planktonic assay (Table 1).

Similar to the overall trend with planktonic cells, $D$. hispida caused significant $(\mathrm{P}<0.05)$ reduction in biofilm density for all bacteria in comparison to the negative control (without $D$. hispida) (Figure 2). Amongst the five bacteria tested, $B$. subtilis and $S$. aureus were the most affected by $D$. hispida, with biofilm densities reducing approximately half or more than half of the negative and positive controls after 24 and $48 \mathrm{~h}$ incubation $(\mathrm{P}<0.05)$. However, for E. coli, P. aeruginosa and K. pneumoniae, $D$. hispida was less effective than gentamicin in reducing their biofilm growth. Furthermore, the fact that free-living K. pneumoniae (Figure 1) could be more effectively eradicated than its biofilm (Figure 2) shows a higher resistance by the bacterium in sessile mode. It is known that these three bacteria ( $E$. coli, $P$. aeruginosa and $K$. pneumonia) produce 'strong' biofilms, resistant to biological and chemical antibiofilm agents, including antibiotics (Donlan and Costerton 2002). Various mechanisms of biofilm resistance have been found in these bacteria, including quorum sensing systems that regulate the expression of resistance-conferring genes and induction of rpoS-mediated stress response, apart from the polymer matrix and biofilm architecture themselves as protective measurement (Mah and O'Toole, 2001; Gellatly and Hancock, 2013; Vital-Lopez et al., 2015). Hence, due to the biofilm higher resistance 
than its planktonic cells, increased concentration of either $D$. hispida or the antibiotic is deemed necessary to eradicate biofilms. Alternatively, the synergy between $D$. hispida and other antimicrobials against biofilms should be explored, as suggested earlier.

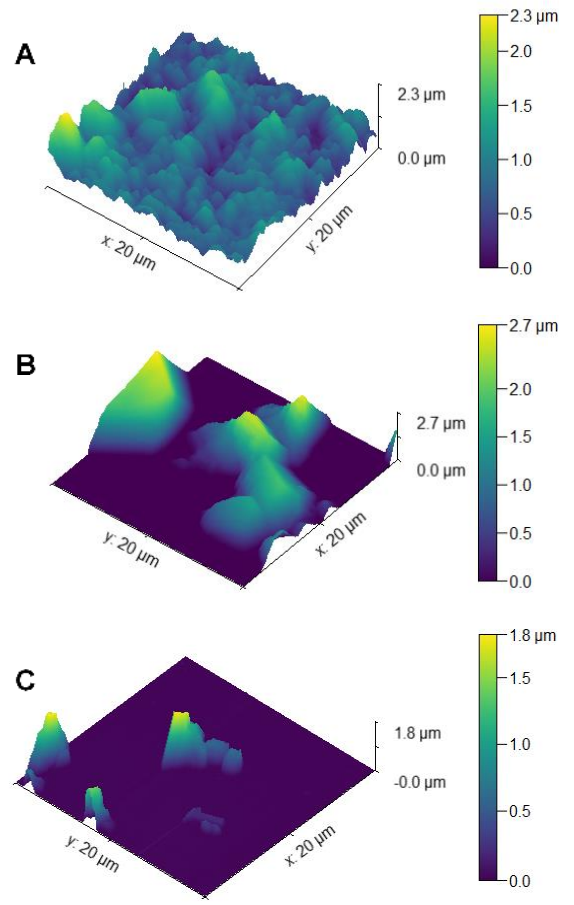

Figure 3: Representative Atomic Force Micrographs (20 $\times 20 \mu \mathrm{m}$ scan) of $S$. aureus biofilms. Treatment: A) without $D$. hispida; B: with $D$. hispida at $2.5 \mathrm{mg} / \mathrm{mL}$ and C: with streptomycin at $150 \mu \mathrm{g} / \mathrm{mL}$.

The action of $D$. hispida starch against biofilms was further explored through AFM imaging, using $S$. aureus as the bacterial model and streptomycin as the antibiotic control (Figure 3). The micrographs showed full surface coverage and relative evenness of $S$. aureus biofilm without any treatment (Figure $3 \mathrm{~A}$ ), in contrast to the increasing sparseness of the biofilm with $D$. hispida (Figure $3 \mathrm{~B}$ ) and antibiotic (Figure $3 \mathrm{C}$ ). Treatment with $D$. hispida markedly caused a reduction in biofilm volume $\left(193 \pm 12 \mu \mathrm{m}^{3}\right)$ compared to the negative control (312 \pm $\left.20 \mu \mathrm{m}^{3}\right)$. However, treatment with streptomycin reduced the biofilm volume even further $\left(29 \pm 7 \mu \mathrm{m}^{3}\right)$ than treatment with $D$. hispida, contrasting the trend obtained in the biofilm quantification assay (where the antibiotic performed weaker than $D$. hispida) shown in Figure 2. This was likely due to the change of antibiotic used (i.e. streptomycin instead of gentamicin), indicating varied responses from $S$. aureus biofilm against different antibiotics. Interestingly, despite the volume reduction and lesser surface coverage, treatment with $D$. hispida caused the biofilm to appear taller (Figures 3 and 4) compared to the negative control, with the average height of $1.72 \pm 0.5$ and $0.71 \pm 0.2 \mu \mathrm{m}$, respectively. Biofilm height distribution (Figure 4) also indicates that biofilm with $D$. hispida generated peaks of between 1.6-2 $\mu \mathrm{m}$, which were not found in either control with a lower height average. This behaviour may signify a mechanism of $S$. aureus biofilm resistance towards $D$. hispida at $2.5 \mathrm{mg} / \mathrm{mL}$ by congregating more cells and/or extracellular polymer matrix to form larger and taller biofilm clusters in order to protect itself from $D$. hispida biocidal activity. However it is not known whether cells in the biofilm with $D$. hispida were mostly alive or dead, or if $D$. hispida was able to penetrate into the deeper area of biofilm through voids and channels, warranting further investigation into the plant antibiofilm mechanism of action. It is anticipated that a higher concentration of $D$. hispida would lessen biofilm formation further, causing a similar reduction effect observed in the antibiotic treatment (Figure 3C).

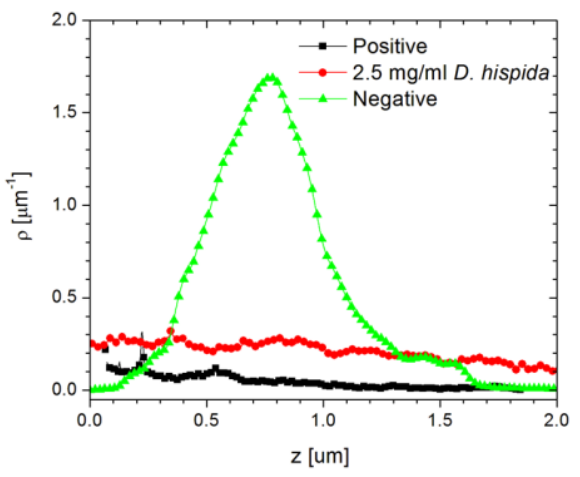

Figure 4: Height (z) distribution $(\mu \mathrm{m})$ of $S$. aureus biofilms with $D$. hispida at $2.5 \mathrm{mg} / \mathrm{mL}$ (red circle), positive control (streptomycin) at $150 \mu \mathrm{g} / \mathrm{mL}$ (black square) and negative (no treatment) control (green triangle) obtained from the AFM scans.

\section{CONCLUSION}

This study shows that $D$. hispida significantly inhibited planktonic cell growth and biofilm density of bacteria compared to untreated samples. However, compared to antibiotics, only certain bacteria were significantly affected by $D$. hispida, implying a "moderate" action of this plant as an antimicrobial. Thus, we suggest that $D$. hispida is used concurrently with another antimicrobial agent to enhance killing efficiency and further reduce the chances of resistance from occurring. Additionally, treatment with $D$. hispida at a certain concentration resulted in taller and larger biofilm clusters despite the reduced overall volume. This finding was in contrast to biofilms without $D$. hispida, highlighting unique biofilm mechanism of resistance against the plant. Overall, this study provides further insights into the effectiveness of traditional plants as a natural alternative in combating infectious diseases and antimicrobial resistance.

\section{ACKNOWLEDGEMENTS}

This work was supported by Geran Galakan Penyelidik Muda, Universiti Kebangsaan Malaysia (UKM) (GGPM- 
2016-017). We are grateful to Dee Chang $\mathrm{Fu}$ and $\mathrm{Ng}$ Kong Eng from the Institute of Microengineering and Nanoelectronics (IMEN) UKM and Weng Lee Lim from WlimLab Sdn. Bhd. for their help on AFM imaging and data analyses.

\section{REFERENCES}

Ajiboye, T. O., Skiebe, E. and Wilharm, G. (2018). Phenolic acids potentiate colistin-mediated killing of Acinetobacter baumannii by inducing redox imbalance. Biomedicine and Pharmacotherapy 101, 737-744.

Araya-Cloutier, C., Vincken, J. P., van Ederen, R., den Besten, H. M. W. and Gruppen, H. (2018). Rapid membrane permeabilization of Listeria monocytogenes and Escherichia coli induced by antibacterial prenylated phenolic compounds from legumes. Food Chemistry 240, 147-155.

Azman, I., Mutalib, S. A., Yusoff, S. F., Fazry, S., Noordin, A., Kumaran, M. and Mat Lazim, A. (2015). Novel Dioscorea hispida starch-based hydrogels and their beneficial use as disinfectants. Journal of Bioactive and Compatible Polymers 31(1), 42-59.

Bhamarapravati, S., Penland, S. L. and Mahady, G. B. (2003). Extracts of spice and food plants from Thai traditional medicine inhibit the growth of the human carcinogen Helicobacter pylori. In Vivo 17, 541-544.

Bjarnsholt, T. (2013). The role of bacterial biofilms in chronic infections. Acta Pathologica, Microbiologica, et Immunologica Scandinavica 121, 1-58.

Carvalho, R., Carollo, C., de Magalhães, J., Palumbo, J., Boaretto, A. and Nunes e Sá, I. (2018). Antibacterial and antifungal activities of phenolic compound-enriched ethyl acetate fraction from Cochlospermum regium (mart. Et. Schr.) Pilger roots: Mechanisms of action and synergism with tannin and gallic acid. South African Journal of Botany 114, 181187.

Donlan, R. M. and Costerton, J. W. (2002). Biofilms: survival mechanisms of clinically relevant microorganisms. Clinical Microbiology Review 15, 167-193.

Gellatly, S. L. and Hancock, R. E. W. (2013). Pseudomonas aeruginosa: New insights into pathogenesis and host defenses. Pathogens and Disease 67, 159-173.

Greenberg, M., Dodds, M. and Tian, M. (2008). Naturally occurring phenolic antibacterial compounds show effectiveness against oral bacteria by a quantitative structure-activity relationship study. Journal of Agricultural and Food Chemistry 56(23), 11151-11156.

Høiby, N., Bjarnsholt, T., Givskov, M., Molin, S. and Ciofu, O. (2010). Antibiotic resistance of bacterial biofilms. International Journal of Antimicrobial Agents 35(4), 322-332.

Hou, W., Lee, M., Chen, H., Liang, W., Han, C., Liu, Y. and Lin, Y. (2001). Antioxidant activities of dioscorin, the storage protein of yam (Dioscorea batatas Decne) tuber. Journal of Agricultural and Food Chemistry 49(10), 4956-4960.

Hron, P., Ŝlechtová, J., Smetana, K., Dvořánková, B. and Lopour, P. (1997). Silicone rubber-hydrogel composites as polymeric biomaterials: IX. Composites containing powdery polyacrylamide hydrogel. Biomaterials 18, 1069-1073.

Lister, J. L. and Horswill, A. R. (2014). Staphylococcus aureus biofilms: Recent developments in biofilm dispersal. Frontiers in Cellular and Infection Microbiology 4, 178.

Mah, T. C. and O'Toole, G. A. (2001). Mechanisms of biofilm resistance to antimicrobial agents. Trends in Microbiology 9(1), 34-39.

Mat Lazim, A., Azman, I., Yusoff, S. F. M., Hassan, N. I., Fazry, S. and Mat Arip, M. N. (2016). Synthesis and characterization of Dioscorea hispida sp. tuber starch-polyacrylamide wood coating and its facile inhibitory towards Pycnoporus sanguineus and Coptotermes curvignathus. Progress in Organic Coatings 99, 182-190.

Miah, M., Das, P., Ibrahim, Y., Shajib, M. and Rashid, M. (2018). In vitro antioxidant, antimicrobial, membrane stabilization and thrombolytic activities of Dioscorea hispida Dennst. European Journal of Integrative Medicine 19(April), 121-127.

Nečas, D. and Klapetek, P. (2012). Gwyddion: An opensource software for SPM data analysis. Central European Journal of Physics 10(1), 181-188.

O'Toole, G. A. (2011). Microtiter dish biofilm formation assay. Journal of Visualized Experiments 30, 47.

Ohizumi, Y., Gaidamashvili, M., Ohwada, S., Matsuda, K., Kominami, J., Nakamura-Tsuruta, S., Hirabayashi, J., Naganuma, T., Ogawa, T. and Muramoto, K. (2009). Mannose-binding lectin from yam (Dioscorea batatas) tubers with insecticidal properties against Helicoverpa armigera (Lepidoptera: Noctuidae). Journal of Agricultural and Food Chemistry 57(7), 2896-2902.

Otake, T., Mori, H., Morimoto, M., Ueba, N., Sutardjo, S., Kusumoto, I. T., Hattori, M. and Namba, T. (1995). Screening of Indonesian plant extracts for anti-human immunodeficiency virus-type 1 (HIV-1) activity. Phytotherapy Research 9, 6-10.

Taylor, T. J., Seitz, E. P., Fox, P., Fischler, G. E., Fuls, J. L. and Weidner, P. L. (2004). Physicochemical factors affecting the rapid bactericidal efficacy of the phenolic antibacterial triclosan. International Journal of Cosmetic Science 26(3), 111-116.

Unemo, U. and Jensen, J. S. (2017). Antimicrobialresistant sexually transmitted infections: Gonorrhoea and Mycoplasma genitalium. Nature Reviews Urology 14, 139-152.

Vital-Lopez, F. G., Reifman, J. and Wallqvist, A. (2015). Biofilm formation mechanisms of Pseudomonas aeruginosa predicted via genome-scale kinetic models of bacterial metabolism. Public Library of Science Computational Biology 11(10), 1-24. 